\title{
A influência da incompatibilidade do macho de rosca na resistência à extração do parafuso pedicular*
}

\section{The Influence of Thread Tap Mismatch on Pedicle Screw Pullout Strength}

\author{
Rômulo Pedroza Pinheiro ${ }^{10}$ Raffaello de Freitas Miranda10 Antonio Carlos Shimano ${ }^{10}$ \\ Thibault Chandanson ${ }^{2}$ Keri George ${ }^{3 \odot}$ Helton L.A. Defino $\left.{ }^{1(}\right)$
}

\footnotetext{
1 Setor de Cirurgia da Coluna, Departamento de Ortopedia e Anestesiologia, Faculdade de Medicina de Ribeirão Preto, Universidade de São Paulo, Ribeirão Preto, SP, Brasil

2 Département Génie Mécanique et Conception, Université de Technologie de Belfort, Montbéliard, França

${ }^{3}$ Departamento de Bacharelado em Enfermagem, University of Calgary, Alberta, Canadá
}

Rev Bras Ortop 2022;57(2):327-333.

\author{
Endereço para correspondência Rômulo Pedroza Pinheiro, University \\ of São Paulo, Ribeirão Preto Medical School, Department of \\ Orthopedics and Anesthesiology, Av. Bandeirantes, 3900, Monte \\ Alegre, Ribeirão Preto, SP, 14049900, Brasil \\ (e-mail: romulopinheiro@usp.br).
}

\section{Resumo}

Palavras-chave

- fusão vertebral

- parafusos pediculares

- parafusos ósseos

- fenômenos biomecânicos/ fisiologia
Objetivo Nosso objetivo foi estudar a resistência à extração "in vitro" do parafuso SpineGuard/Zavation Dynamic Surgical Guidance Z-Direct (Parafuso DSG Guia Cirúrgico Dinâmico, SpineGuard Inc, Boulder, Colorado, USA), um parafuso projetado para ser inserido utilizando a técnica de inserção direta.

Métodos Os parafusos DSG de 5,5 e $6,5 \mathrm{~mm}$ foram introduzidos em blocos de poliuretano com densidade de $10 \mathrm{PCF}\left(0,16 \mathrm{~g} / \mathrm{cm}^{3}\right)$. De acordo com o grupo experimental, os parafusos foram inseridos sem um orifício piloto, com um orifício piloto sem o macheamento, com macheamento e com macheamento linha a linha. Os testes de extração do parafuso foram realizados em uma máquina de teste universal, após a inserção do parafuso em blocos de poliuretano.

Resultados Os parafusos inseridos diretamente nos blocos de poliuretano sem o orifício piloto e o macheamento mostraram uma resistência à extração estatisticamente maior. A inserção do parafuso sem o macheamento ou com o macho de menor diâmetro aumenta a resistência à extração do parafuso em comparação com o macheamento linha a linha.

Conclusão O parafuso DSG apresentou a maior resistência à extração após a inserção sem o orifício piloto e o macheamento.
Trabalho desenvolvido no Laboratório de Bioengenharia da Faculdade de Medicina de Ribeirão Preto da Universidade de São Paulo, Ribeirão Preto, SP, Brasil.

\section{recebido}

05 de Novembro de 2020

aceito

08 de Março de 2021

Publicado on-line

de Janeiro 21, 2022
DOI https://doi.org/ 10.1055/s-0041-1732390. ISSN 0102-3616. (c) 2022. Sociedade Brasileira de Ortopedia e Traumatologia. All rights reserved.

This is an open access article published by Thieme under the terms of the Creative Commons Attribution-NonDerivative-NonCommercial-License, permitting copying and reproduction so long as the original work is given appropriate credit. Contents may not be used for commercial purposes, or adapted, remixed, transformed or built upon. (https://creativecommons.org/ licenses/by-nc-nd/4.0/)

Thieme Revinter Publicações Ltda., Rua do Matoso 170, Rio de Janeiro, RJ, CEP 20270-135, Brazil 


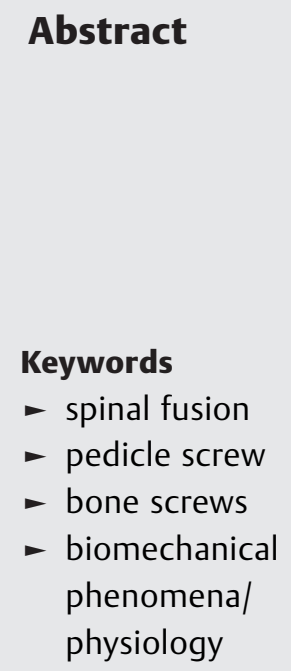

Objective We aimed to study the "in vitro" pullout strength of SpineGuard/Zavation Dynamic Surgical Guidance Z-Direct Screw (DSG Screw, SpineGuard Inc, Boulder, Colorado, USA), a screw designed to be inserted using a direct insertion technique. Methods Dynamic Surgical Guidance Screws of 5.5 and $6.5 \mathrm{~mm}$ were introduced into polyurethane blocks with a density of $10 \operatorname{PCF}\left(0,16 \mathrm{~g} / \mathrm{cm}^{3}\right)$. According to the experimental group, screws were inserted without pilot hole, with pilot without tapping, undertapping and line-to-line tapping. Screw pullout tests were performed using a universal test machine after screw insertion into polyurethane blocks.

Results Screws inserted directly into the polyurethane blocks without pilot hole and tapping showed a statistically higher pullout strength. Insertion of the screw without tapping or with undertapping increases the pullout screw strength compared with lineto-line tapping.

Conclusion Dynamic Surgical Guidance Screw showed the highest pullout strength after its insertion without pilot hole and tapping.

\section{Introdução}

A construção com parafuso pedicular é, atualmente, o método mais usado para a fixação interna da coluna torácica e lombar. ${ }^{1,2}$ A utilidade clínica da construção com o parafuso pedicular está respaldada pela alta taxa de fusão, correção de deformidade e resultados clínicos. ${ }^{1-3} \mathrm{~A}$ acurácia média dos parafusos pediculares inseridos à mão livre ou assistida por fluoroscopia é de $85,1 \%$, sendo que com a técnica de navegação cirúrgica o índice alcança $95 \% .{ }^{4}$ Os principais problemas relacionados às fixações pediculares incluem as propriedades mecânicas dos parafusos pediculares, a acurácia e o uso da radiação intraoperatória para a colocação. Falhas, como o afrouxamento do parafuso, ainda ocorrem, apesar dos avanços tecnológicos, sendo relatadas em 0,6 a $11 \%{ }^{5,6}$ A colocação e a exposição à radiação do cirurgião, principalmente em procedimentos minimamente invasivos, são as desvantagens da fixação do parafuso pedicular. ${ }^{3-5} \mathrm{~A}$ exposição do cirurgião à radiação durante uma cirurgia com parafuso pedicular toracolombar assistida por fluoroscopia é 10 a 12 vezes maior em comparação com outros procedimentos cirúrgicos não espinhais assistidos pela técnica fluoroscópica., ${ }^{2,7,8}$

O dispositivo Pediguard Threaded Device (PTD) foi desenvolvido para preparar o orifício piloto na vértebra, contornando os problemas relacionados à inserção do parafuso pedicular. $\mathrm{O}$ dispositivo PTD é um instrumento de perfuração com desenho de rosca e um sensor na extremidade, com a finalidade de perfurar o orifício piloto, seguido diretamente pela inserção do parafuso, reduzindo, assim, as etapas cirúrgicas e a exposição à radiação, além de proporcionar uma maior precisão. ${ }^{9-11}$

O PTD é um instrumento de perfuração com a extremidade rosqueada, disponível em vários tamanhos (4,0, 4,5 e 5,5 mm) com diferentes desenhos de rosca. Ele tem o propósito de otimizar as etapas cirúrgicas, mantendo a precisão do preparo pedícular para a colocação do parafuso. $\mathrm{O}$ uso do dispositivo PTD permite preparar o orifício piloto do pedículo, com o objetivo de fornecer uma adequada tração mecânica do parafuso, melhorando a precisão do parafuso pedicular com a utilização da impedância em sua extremidade.
Atualmente, os cirurgiões da coluna realizam o macheamento no orifício piloto antes da inserção do parafuso pedicular. ${ }^{10} \mathrm{O}$ macheamento no orifício piloto permite a inspeção das paredes pedículares antes da inserção do parafuso, orientando a inserção do parafuso no pedículo. ${ }^{11}$ Uma rosca na superfície interna do orifício piloto é produzida pelo macho, criando uma superfície fêmea para o parafuso pedicular. Embora o uso de macho com o mesmo diâmetro do parafuso pedicular resulte em uma compatibilidade perfeita, isso reduz a resistência à extração do parafuso, não sendo recomendado, principalmente em ossos osteoporóticos. ${ }^{3,4,12} \mathrm{~A}$ resistência à extração do parafuso está relacionada à tração do parafuso e à estabilidade biomecânica do sistema de fixação pedícular. O uso de um macho $1 \mathrm{~mm}$ menor do que o diâmetro do parafuso (diâmetro externo) aumenta a resistência à extração do parafuso pedicular, sendo que o macheamento é comumente usado para a colocação do parafuso pedicular. ${ }^{10}$

Foi relatado que o macheamento com passo incongruente (distância longitudinal entre a crista da rosca) reduz a resistência à extração do parafuso pedicular. ${ }^{10} \mathrm{O}$ uso de um macho com passo diferente do parafuso pedicular não permite que a rosca do parafuso se ajuste à ranhura précortada para o macho. O que motivou a realização do presente estudo foi a potencial preocupação levantada com o uso do dispositivo PTD para a inserção dos parafusos pediculares com diferentes passos.

O objetivo do presente estudo foi avaliar experimentalmente a influência do macheamento do orifício piloto com o dispositivo PTD e o parafuso com passo diferente do macho. Testamos a hipótese de que o macheamento com roscas congruentes e incongruentes apresenta um efeito semelhante na resistência à extração do parafuso.

\section{Métodos}

Foram utilizados como corpos de prova para a introdução dos parafusos e a realização dos ensaios mecânicos de extração 105 de poliuretano de $8 \mathrm{~cm}$ de altura, $5 \mathrm{~cm}$ de largura e $5 \mathrm{~cm}$ 
de comprimento, com densidade de 10 PCF $\left(0,16 \mathrm{~g} / \mathrm{cm}^{3}\right)$ (Nacional Ltda, Santana de Parnaíba, SP, Brasil). Foi feito um pré-furo de $40 \mathrm{~mm}$ de profundidade no centro do bloco de poliuretano, com o uso de uma broca de $2,7 \mathrm{~mm}$. Os parafusos foram inseridos diretamente na espuma, após o macheamento de acordo com o grupo experimental. Foram realizados três tipos de macheamento: 1-macheamento linha a linha (macho com mesmo passo e diâmetro externo do parafuso), 2-macheamento com passo congruente (macho com mesmo passo e diâmetro externo $1 \mathrm{~mm}$ menor do que o do parafuso) e 3-macheamento com passo incongruente (macho com passo diferente e/ou número de derivação diferente e diâmetro externo $1 \mathrm{~mm}$ menor que parafuso).

O macho com passo congruente foi um macho fornecido por empresas como parte do conjunto de instrumentação. Um passo congruente significa que o passo da rosca do macho é o mesmo do parafuso. Foram utilizados machos congruentes do mesmo diâmetro externo do parafuso (5,5 e $6,5 \mathrm{~mm}$ ) (macho linha a linha) ou $1 \mathrm{~mm}$ menor (4,5 e $5,5 \mathrm{~mm}$ ) (macheamento), de acordo com o grupo experimental.

Foi usado o dispositivo PTD com diferentes diâmetros e passos no grupo experimental de macheamento com passo incongruente. Foram usados 2 dispositivos PTD de $4,5 \mathrm{~mm}$ (Ped D1TA0011 e Ped D1TA0013) como macheamento com passo incongruente em parafusos de $5,5 \mathrm{~mm}$. O macho D1TA0011 tem passo de $2,9 \mathrm{~mm}$ com desenho de derivação dupla, e o D1TA0013 tem passo de $2,5 \mathrm{~mm}$ com desenho de derivação dupla. Foi usado um PTD de 5,5 mm (D1TA0001) como macheamento com passo incongruente em um parafuso de $6,5 \mathrm{~mm}$. O macho D1TA0001 possui um passo de 2,8 mm com um desenho de derivação simples. Em comparação com os respectivos machos do fabricante, os dispositivos com rosca Pediguard também possuem uma extremidade com uma parte sem rosca $(\sim 10 \mathrm{~mm})$ para redirecionamento (- Figura $\mathbf{1}$ ).

Foram utilizados 3 tipos diferentes de parafusos pediculares de 5,5 e 6,5 mm de diâmetro externo, com diferentes tipos ou desenhos de diâmetros e passos diferentes. Os

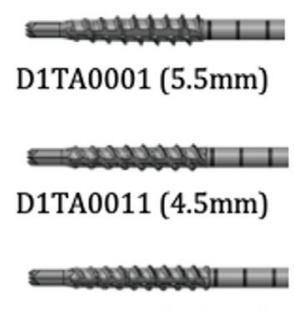

D1TA0013 $(4.5 \mathrm{~mm})$

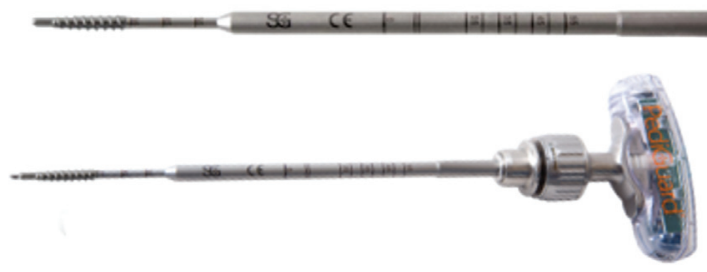

Fig. 1 Broca roscada PediGuard usada no estudo.

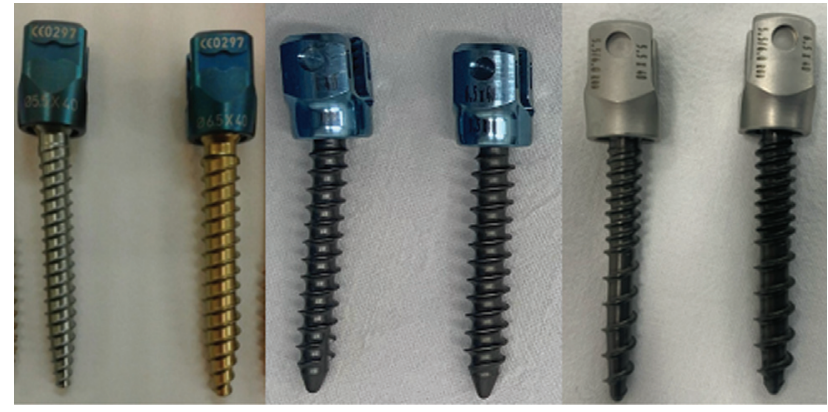

Fig. 2 Parafusos de 5,5 mm e 6,5 mm de diâmetro externo.

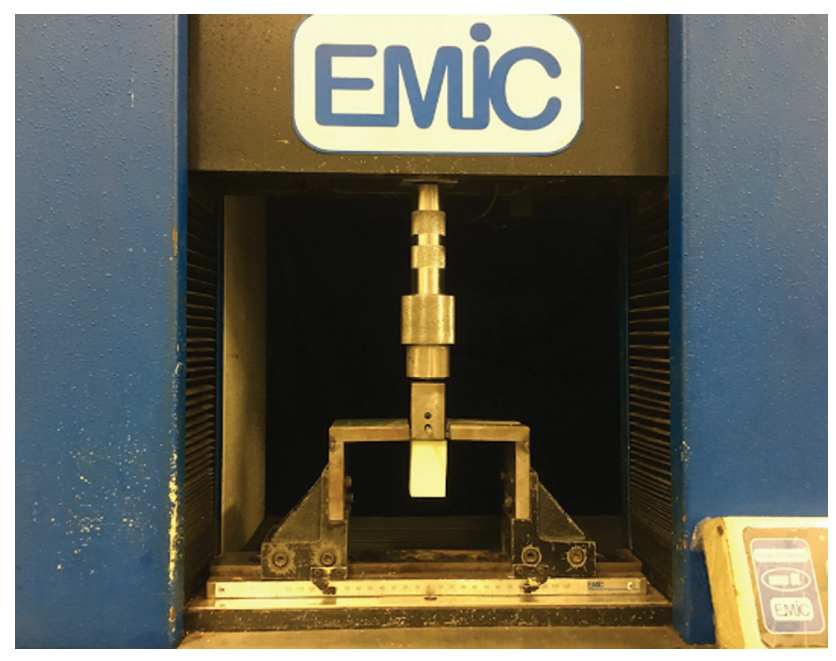

Fig. 3 Máquina de teste universal EMIC.

parafusos com diâmetro afunilado e passo homogêneo (Legacy-Medtronic), os parafusos com alma e roscas de dois tipos (Solera-Medtronic) e os com alma cônica, em caso de roscas homogêneas (Revere-Globus Minneapolis, MN, USA) (-Figura 2).

O grupo experimental foi formado de acordo com o tipo de parafuso (diâmetro externo e fabricante) e a preparação do orifício piloto. Cada grupo experimental foi formado por cinco blocos de poliuretano.

Após a inserção do parafuso, foi avaliada a resistência à extração em máquina de ensaio universal (EMIC-DL10000, São José dos Pinhais, PR, Brasil). Uma haste foi fixada à cabeça do parafuso, sendo aplicada uma força de extração de forma vertical (-Figura 3). Foi aplicada a força de extração a uma velocidade de $2,0 \mathrm{~mm} / \mathrm{min}$ até que o parafuso fosse retirado do bloco de poliuretano (-Figura 1).

\section{Análise Estatística}

Foi utilizado o teste não paramétrico de Kruskal-Wallis para comparar a resistência à extração entre os diferentes parafusos. O nível de significância foi estabelecido em $5 \%$ ( $p \leq$ 0,05 ). Com o propósito de definir melhor essas diferenças, foi realizado o pós-teste de comparações múltiplas de Dunn e comparado com valores de $p$ menores do que o nível de significância adotado $(0,05)$, sendo esse o indicativo de uma diferença entre os grupos. 
Tabela 1 Resistencia à extração do parafuso pedicular Legacy $5,5 \mathrm{~mm}$

\begin{tabular}{|l|l|}
\hline Macho & Resistência à Extração (N) \\
\hline Macho Legacy 5,5 & $587,9 \pm 18,19^{(*)(* *)}$ \\
\hline Macho Legacy 4,5 & $549,0 \pm 21,92^{(*)}$ \\
\hline Ped 4,5 D1TA 11 & $544,2 \pm 3,176^{(* *)}$ \\
\hline Ped 4,5 D1TA 13 & $531,0 \pm 34,03$ \\
\hline
\end{tabular}

Os asteriscos $\left({ }^{*}\right)$ e $\left({ }^{* *}\right)$ indicam uma diferença estatística.

\section{Resultados}

Os resultados da resistência à extração do parafuso nos grupos experimentais de acordo com o macheamento estão ilustrados nas Tabelas e Figuras.

Não houve diferença estatística entre a resistência à extração dos parafusos de 5,5 e 6,5 $\mathrm{mm}$ da marca Legacy (-Tabela 1 e -Figura 4), usando o macheamento com passo congruente ou o macheamento com passo incongruente. $\mathrm{O}$ macheamento com o parafuso incongruente mostrou resistência à extração estatisticamente inferior em comparação com o macheamento linha a linha. ( $p=0,0089)$.

O parafuso Legacy de 5,5 mm apresentou menor resistência à extração com a utilização do macheamento linha a linha em comparação ao macheamento com passo congruente e incongruente. Foi observada diferença estatística entre o macheamento com o passo congruente e incongruente, com o uso do macho D1TA0011 $(p<0,05)$, em comparação com macheamento linha-a-linha. Nenhuma diferença estatística foi observada entre macheamento com a utilização do passo incongruente com o macho D1TA0013 (- Tabela 2 e - Figura 5 ).

Não houve diferença estatística entre a resistência à extração dos parafusos de 5,5 e 6,5 mm da marca Solera (-Tabelas 3, 4 e - Figura 6,7) usando o macheamento com o passo congruente ou o macheamento com o passo incongruente.

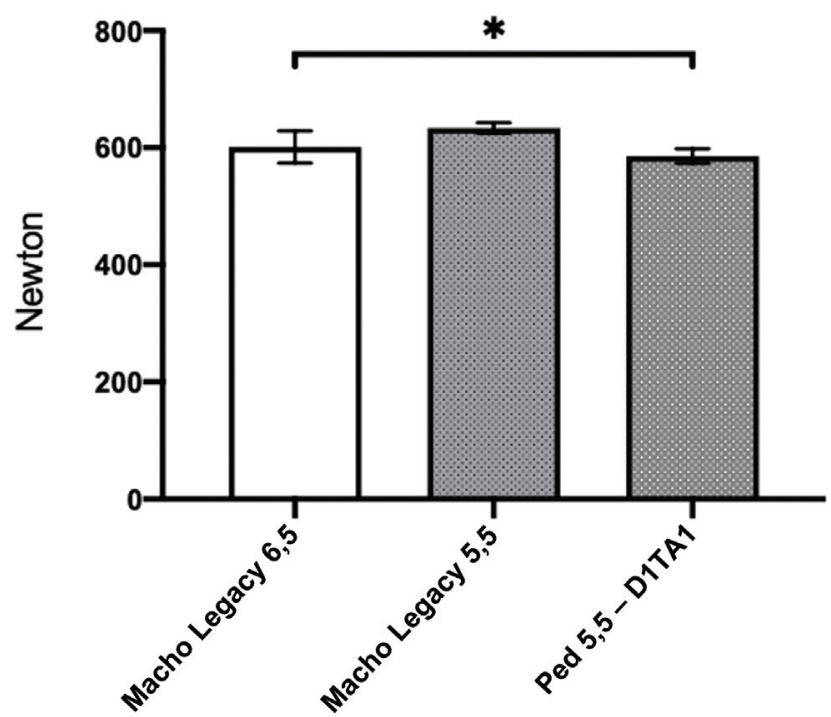

Fig. 4 Resistencia à extração do parafuso pedicular Legacy de $6,5 \mathrm{~mm}$.
Tabela 2 Resistencia à extração do parafuso pedicular Legacy $6,5 \mathrm{~mm}$

\begin{tabular}{|l|l|}
\hline Macho & Resistência à Extração (N) \\
\hline Macho Legacy 6,5 & $601,5 \pm 27,40^{(*)}$ \\
\hline Macho Legacy 5,5 & $633,6 \pm 9,311$ \\
\hline Ped 5,5 - D1TA 1 & $585,8 \pm 12,43^{(*)}$ \\
\hline
\end{tabular}

Os asteriscos $\left({ }^{*}\right)$ indicam uma diferença estatística.

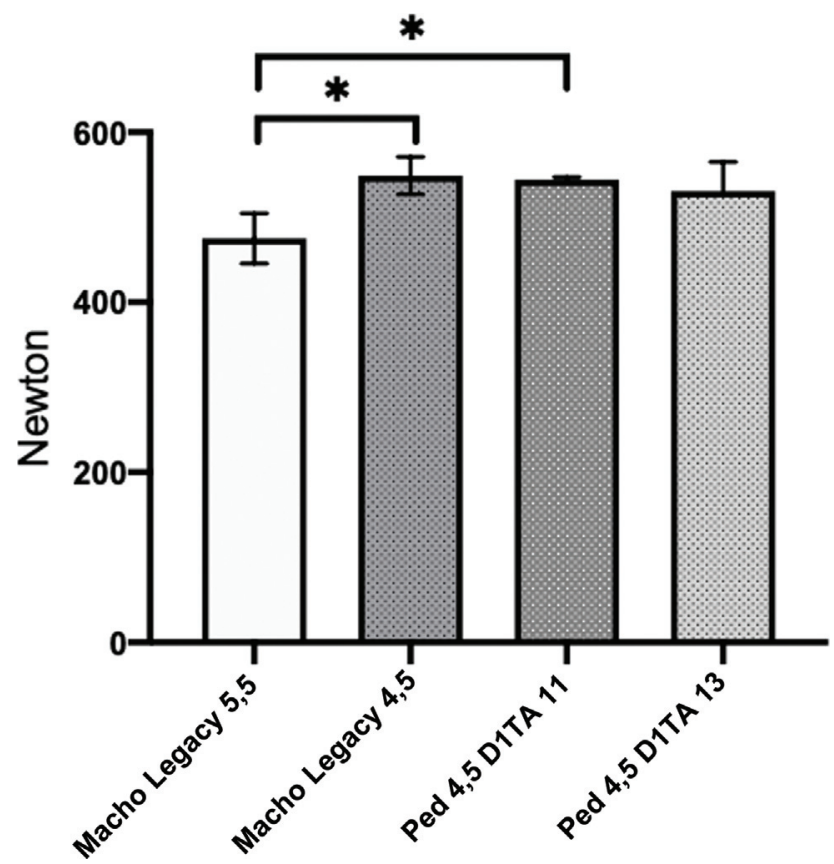

Fig. 5 Resistencia à extração do parafuso pedicular Legacy de $5,5 \mathrm{~mm}$.

Tabela 3 Resistencia à extração do parafuso pedicular Solera $5,5 \mathrm{~mm}$

\begin{tabular}{|l|l|}
\hline Macho & Resistência à Extração (N) \\
\hline Macho Solera 5,5 & $541,3 \pm 18,19$ \\
\hline Macho Solera 4,5 & $546,6 \pm 42,46$ \\
\hline Ped 4,5 D1TA 11 & $501,1 \pm 29,16$ \\
\hline Ped 4,5 D1TA 13 & $513,8 \pm 14,56$ \\
\hline
\end{tabular}

Tabela 4 Resistencia à extração do parafuso pedicular Solera $6,5 \mathrm{~mm}$

\begin{tabular}{|l|l|}
\hline Macho & Resistência à Extração (N) \\
\hline Macho Solera 6,5 & $581,3 \pm 42,10^{(*)}$ \\
\hline Macho Solera 5,5 & $662,8 \pm 48,32^{(*)}$ \\
\hline Ped 5,5 - D1TA 1 & $607,8 \pm 20,27$ \\
\hline
\end{tabular}

Os asteriscos $\left({ }^{*}\right)$ indicam uma diferença estatística. 


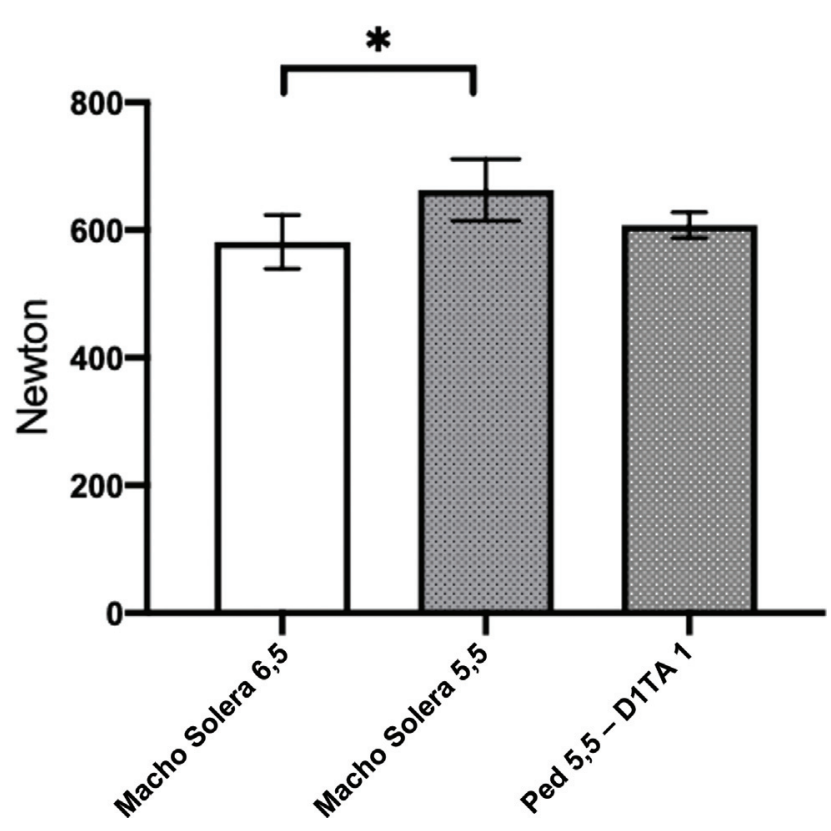

Tabela 5 Resistencia à extração do parafuso pedicular Globus $5,5 \mathrm{~mm}$

\begin{tabular}{|l|l|}
\hline Macho & Resistência à Extração (N) \\
\hline Macho Globus 5,5 & $587,9 \pm 18,19$ \\
\hline Macho Globus 4,5 & $607,2 \pm 21,88$ \\
\hline Ped 4,5 D1TA 11 & $625,7 \pm 24,54^{(*)}$ \\
\hline Ped 4,5 D1TA 13 & $581,6 \pm 9,776^{(*)}$ \\
\hline
\end{tabular}

Os asteriscos $\left({ }^{*}\right)$ indicam uma diferença estatística.

Tabela 6 Resistencia à extração do parafuso pedicular Globus $6,5 \mathrm{~mm}$

\begin{tabular}{|l|l|}
\hline Macho & Resistência à Extração (N) \\
\hline Macho Globus 6,5 & $667,3 \pm 14,36$ \\
\hline Macho Globus 5,5 & $678,0 \pm 21,03$ \\
\hline Ped 5,5 - D1TA 1 & $652,9 \pm 16,17$ \\
\hline
\end{tabular}

Fig. 6 Resistencia à extração do parafuso pedicular Solera $6,5 \mathrm{~mm}$.
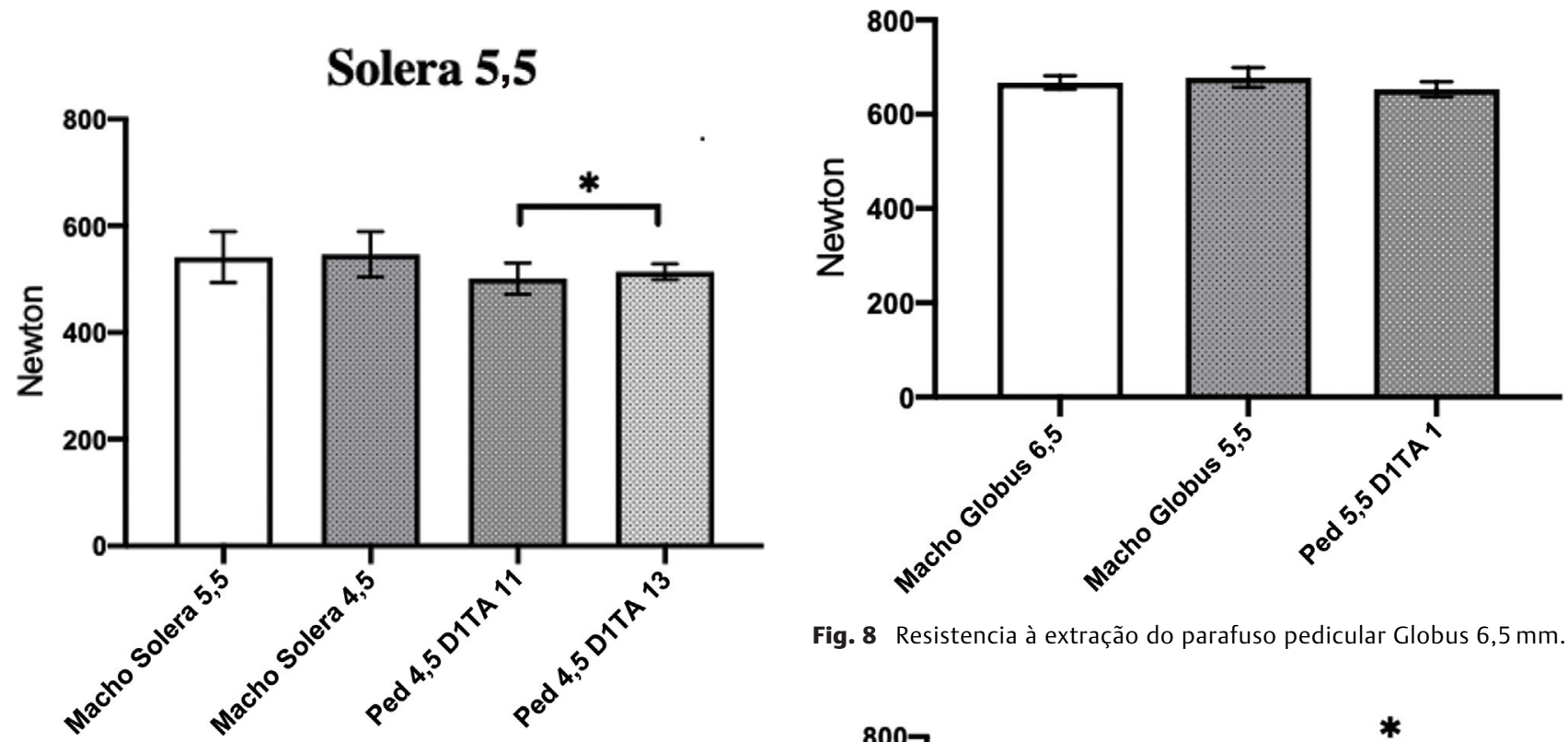

Fig. 8 Resistencia à extração do parafuso pedicular Globus $6,5 \mathrm{~mm}$.

Fig. 7 Resistencia à extração do parafuso pedicular Solera 5,5 mm.

Não houve diferença estatística entre a resistência à extração dos parafusos de 5,5 e $6,5 \mathrm{~mm}$ da marca Revere (Globus) (-Tabelas 5,6 e -Figura 8,9) usando o macheamento com o passo congruente ou o macheamento com 0 passo incongruente.

\section{Discussão}

Nossos resultados apoiam a hipótese de que o macheamento do orifício piloto com passo incongruente não reduz a resistência à extração do parafuso em comparação com o macheamento com passo congruente, com todas as modalidades de parafusos pediculares testadas. O efeito do

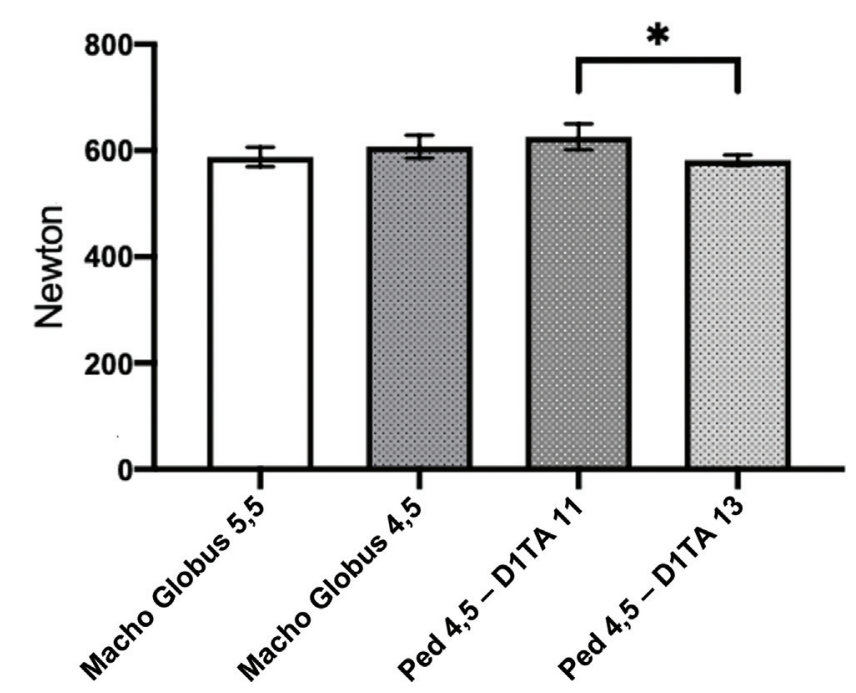

Fig. 9 Resistencia à extração do parafuso pedicular Globus 5,5 mm. 
macheamento não foi uniforme com os três tipos de parafusos pedículares usados no estudo.

Embora os parafusos pediculares sejam amplamente utilizados em cirurgia da coluna vertebral, ainda existem problemas devido à fixação insuficiente da interface entre os parafusos e o osso. ${ }^{13} \mathrm{~A}$ taxa relatada de afrouxamento do parafuso pedicular é de 0,6 a $11 \% .^{14,15}$ É importante evitar o afrouxamento do parafuso, sob o ponto de vista da biomecânica, assegurando, assim, a estabilidade mecânica nas vértebras. Várias estratégias foram feitas no desenho do implante e na preparação do orifício piloto a fim de melhorar a fixação na interface do parafuso ósseo, com o objetivo de melhorar o afrouxamento do parafuso. ${ }^{10,16}$

A preparação do orifício piloto é a única variável que pode ser controlada durante o ato cirúrgico por intermédio dos cirurgiões. Foram relatados os parâmetros relacionados à preparação do orifício piloto, como diâmetro, modo de preparação, macheamento e a inserção repetitiva do parafuso. ${ }^{10,17-19}$ Foi realizada a otimização do orifício piloto com o objetivo de melhorar a ancoragem mecânica dos parafusos nas vértebras. ${ }^{20}$

O uso do dispositivo PTD com passo diferente, em comparação com os desenhos dos parafusos utilizados no presente estudo, não reduziu a resistência à extração de todos os tipos de parafusos usados no estudo. O efeito do macheamento com passo incongruente observado em nosso estudo não se correlacionou com os resultados relatados por Bohl et $\mathrm{al}^{10}$ que relataram a redução da resistência à extração do parafuso com macheamento de passo incongruente em comparação com o macheamento com passo congruente. 0 macheamento com diâmetro menor do que o do parafuso possui diâmetro interno e externo menor que o parafuso pedicular e, mesmo sendo incongruente, permite o deslocamento radial e a compactação do osso esponjoso pelo núcleo dos parafusos. O desenho do parafuso pode interferir na quantidade máxima de osso compactado, contido dentro da rosca do parafuso que atua sobre a resistência à extração do parafuso. Desde que realizado com menor diâmetro, o passo do macho incongruente por si só não seria capaz de alterar a resistência à extração do parafuso, além de outros fatores que poderiam influir, como o desenho do parafuso. ${ }^{19-22}$

Atualmente, o macheamento do orifício piloto é realizado por cirurgiões da coluna vertebral antes da inserção do parafuso pedicular. ${ }^{10}$ No entanto, os parafusos pediculares pré-macheados do mesmo tamanho reduzem a resistência à extração do parafuso, não sendo recomendados, principalmente em osso osteoporótico. ${ }^{3,12,19} \mathrm{O}$ orifício piloto com menor diâmetro promove o deslocamento radial e a compactação do osso esponjoso através do núcleo do parafuso durante a inserção, resultando em um contato maior ossoparafuso e, consequentemente, em uma maior quantidade de osso dentro da rosca do parafuso. ${ }^{23}$

O macheamento de $1 \mathrm{~mm}$ é considerado seguro e atinge a mesma resistência à extração do parafuso em comparação com os parafusos não macheados que apresentam uma maior resistência à extração. ${ }^{19}$

No campo da cirurgia da coluna vertebral, o macheamento também foi introduzido para permitir a inspeção das paredes do orifício piloto antes da inserção do parafuso, orientando a inserção do parafuso no pedículo. ${ }^{19}$ A precisão da trajetória do parafuso pedicular é aprimorada por meio do macheamento do orifício piloto antes da inserção do parafuso. ${ }^{23} \mathrm{Na}$ prática clínica, também deve ser considerado que o uso do dispositivo PTD reduz o número de etapas cirúrgicas para a inserção do parafuso pedicular, aumenta a precisão e reduz a exposição do cirurgião à radiação. ${ }^{20,24} \mathrm{O}$ dispositivo PTD combina a necessidade de fazer um orifício piloto e um macho em uma etapa, sem reduzir a resistência à extração do parafuso. 25

Deve ser considerada a limitação do modelo experimental utilizado no presente estudo. Não foi realizado o torque de inserção que tem sido usado em muitos estudos experimentais relacionados aos parafusos pediculares. Não há correlação entre o torque de inserção e a resistência à extração, ${ }^{20}$ sendo esse o motivo para não incluir esse tipo de avaliação no presente estudo.

Outra limitação do modelo experimental utilizado no presente estudo é o fato de que foi aplicada apenas a força axial pura e nenhuma força radial. $O$ ensaio foi realizado em uma configuração de parafuso único e não em uma construção completa com hastes ou placas. Os parafusos não foram submetidos à carga lateral, o que influencia na mecânica da interface osso-parafuso.

Por fim, os testes foram realizados em blocos de espuma macia, representando ossos osteoporóticos. Um modelo de maior densidade pode fornecer valores de extração mais elevados e diferentes.

O teste de resistência à extração pode não ser comumente visto em um ambiente clínico; porém, sua simplicidade e reprodutibilidade permitem que seja considerado o método mais eficiente, com o intuito de comparar a ancoragem do parafuso no osso. Este teste é aceito como um bom preditor do desempenho mecânico do parafuso. No entanto, os parafusos pedículares estão sujeitos a uma complexa situação mecanicamente exigente, representada por uma associação de força de torção, curvatura e extração. ${ }^{24,25}$

A simplificação da força estudada aqui pode não representar uma condição clínica real, mas pode fornecer informações úteis quando as comparações são feitas mediante condições controladas. ${ }^{24,25}$ A resistência à extração do parafuso não representa o único mecanismo de falha do parafuso, porém reflete a magnitude da tração do parafuso. ${ }^{25}$

O dispositivo PTD é um instrumento de perfuração com uma extremidade rosqueada, que está disponível em vários tamanhos $(4,0,4,5,5,5 \mathrm{~mm})$ com diferentes desenhos de rosca, sendo usado para otimizar as etapas cirúrgicas, mantendo a precisão para o preparo do pedículo para a colocação do parafuso. Este dispositivo possui uma capacidade de medição de impedância na extremidade. Uma mudança no tom e na cadência do feedback de áudio indica uma alteração no tecido ao redor da extremidade do PTD. Um tom de médio alcance e um sinal de áudio de cadência média pode ser ouvido conforme a sonda avança em direção ao osso esponjoso. Um sinal de áudio de tom e cadência baixos pode ser ouvido conforme a sonda se aproxima da parede cortical do pedículo, sendo esta a primeira indicação de uma possível ruptura. $^{26}$ Tem sido experimental e clinicamente 
demonstrado que a capacidade da sonda com capacidade de impedância na extremidade (sonda Pediguard), melhora a precisão e reduz a exposição do cirurgião à radiação. 6,9-12

Como o cirurgião não pode alterar a qualidade óssea, melhorias no desenho do parafuso e técnicas de inserção são realizadas para aperfeiçoar a fixação do parafuso e o macheamento do orifício piloto. ${ }^{25,27}$ Embora o dispositivo PTD possua um passo incongruente em comparação com os parafusos pediculares usados no presente estudo, o macheamento do orifício piloto com passo incongruente não mostrou nenhuma diferença estatística em comparação com o macheamento com passo congruente.

\section{Conclusão}

O macheamento do orifício piloto com passo congruente ou incongruente não afeta a resistência à extração do parafuso pedicular. A resistência à extração do parafuso pedicular pode ser influenciada por muitos fatores, como o desenho da rosca do parafuso e o uso de um passo incongruente, o qual, por si só, não tem nenhum efeito.

\section{Suporte Financeiro}

Não houve suporte financeiro de fontes públicas, comerciais, ou sem fins lucrativos.

\section{Conflito de Interesses}

Os autores declaram não haver conflito de interesses.

\section{Referências}

1 Aebi M, Etter C, Kehl T, Thalgott J. Stabilization of the lower thoracic and lumbar spine with the internal spinal skeletal fixation system. Indications, techniques, and first results of treatment. Spine (Phila Pa 1976) 1987;12(06):544-551

2 Cho W, Cho SK, Wu C. The biomechanics of pedicle screw-based instrumentation. J Bone Joint Surg Br 2010;92(08):1061-1065

3 Chen LH, Tai CL, Lai PL, et al. Pullout strength for cannulated pedicle screws with bone cement augmentation in severely osteoporotic bone: influences of radial hole and pilot hole tapping. Clin Biomech (Bristol, Avon) 2009;24(08):613-618

4 Mason A, Paulsen R, Babuska JM, et al. The accuracy of pedicle screw placement using intraoperative image guidance systems. J Neurosurg Spine 2014;20(02):196-203

5 Benzel E. Biomechanics of spine stabilization. New York: Thieme; 2001

6 Schatzker J, Horne JG, Sumner-Smith G. The reaction of cortical bone to compression by screw threads. Clin Orthop Relat Res 1975;(111):263-265

7 Derincek A, Wu C, Mehbod A, Transfeldt EE. Biomechanical comparison of anatomic trajectory pedicle screw versus injectable calcium sulfate graft-augmented pedicle screw for salvage in cadaveric thoracic bone. J Spinal Disord Tech 2006;19(04):286-291

8 Oktenoğlu BT, Ferrara LA, Andalkar N, Ozer AF, Sarioğlu AC, Benzel EC. Effects of hole preparation on screw pullout resistance and insertional torque: a biomechanical study. J Neurosurg 2001;94 (1, Suppl):91-96

9 Hsu CC, Chao CK, Wang JL, Hou SM, Tsai YT, Lin J. Increase of pullout strength of spinal pedicle screws with conical core: biomechanical tests and finite element analyses. J Orthop Res 2005;23(04):788-794

10 Bohl DD, Basques BA, Golinvaux NS, et al. Undertapping of Lumbar Pedicle Screws Can Result in Tapping With a Pitch That Differs From That of the Screw, Which Decreases Screw Pullout Force. Spine (Phila Pa 1976) 2015;40(12):E729-E734

11 Kim YY, Choi WS, Rhyu KW. Assessment of pedicle screw pullout strength based on various screw designs and bone densities-an ex vivo biomechanical study. Spine J 2012;12(02):164-168

12 Becker S, Chavanne A, Spitaler R, et al. Assessment of different screw augmentation techniques and screw designs in osteoporotic spines. Eur Spine J 2008;17(11):1462-1469

13 Cameron HU, Jacob R, Macnab I, Pilliar RM. Use of polymethylmethacrylate to enhance screw fixation in bone. J Bone Joint Surg Am 1975;57(05):655-656

14 Inceoglu S, Ferrara L, McLain RF. Pedicle screw fixation strength: pullout versus insertional torque. Spine J 2004;4(05):513-518

15 Daftari TK, Horton WC, Hutton WC. Correlations between screw hole preparation, torque of insertion, and pullout strength for spinal screws. J Spinal Disord 1994;7(02):139-145

16 Zdeblick TA, Kunz DN, Cooke ME, McCabe R. Pedicle screw pullout strength. Correlation with insertional torque. Spine (Phila Pa 1976) 1993;18(12):1673-1676

17 Kuklo TR, Lehman RA Jr. Effect of various tapping diameters on insertion of thoracic pedicle screws: a biomechanical analysis. Spine (Phila Pa 1976) 2003;28(18):2066-2071

18 Chatzistergos PE, Sapkas G, Kourkoulis SK. The influence of the insertion technique on the pullout force of pedicle screws: an experimental study. Spine (Phila Pa 1976) 2010;35(09): E332-E337

19 Carmouche JJ, Molinari RW, Gerlinger T, Devine J, Patience T. Effects of pilot hole preparation technique on pedicle screw fixation in different regions of the osteoporotic thoracic and lumbar spine. J Neurosurg Spine 2005;3(05):364-370

20 Cook SD, Salkeld SL, Stanley T, Faciane A, Miller SD. Biomechanical study of pedicle screw fixation in severely osteoporotic bone. Spine J 2004;4(04):402-408

21 Chen SH, Mo Lin R, Chen HH, Tsai KJ. Biomechanical effects of polyaxial pedicle screw fixation on the lumbosacral segments with an anterior interbody cage support. BMC Musculoskelet Disord 2007;8:28

22 Ponnappan RK, Serhan H, Zarda B, Patel R, Albert T, Vaccaro AR. Biomechanical evaluation and comparison of polyetheretherketone rod system to traditional titanium rod fixation. Spine J 2009; 9(03):263-267

23 Defino HL, Rosa RC, Silva P, et al. The effect of repetitive pilothole use on the insertion torque and pullout strength of vertebral system screws. Spine (Phila Pa 1976) 2009;34(09): 871-876

24 Scifert JL, Sairyo K, Goel VK, et al. Stability analysis of an enhanced load sharing posterior fixation device and its equivalent conventional device in a calf spine model. Spine (Phila Pa 1976) 1999;24 (21):2206-2213

25 Lill CA, Schneider E, Goldhahn J, Haslemann A, Zeifang F. Mechanical performance of cylindrical and dual core pedicle screws in calf and human vertebrae. Arch Orthop Trauma Surg 2006;126 (10):686-694

26 Pfeiffer M, Gilbertson LG, Goel VK, et al. Effect of specimen fixation method on pullout tests of pedicle screws. Spine (Phila Pa 1976) 1996;21(09):1037-1044

27 Pfeiffer FM, Abernathie DL, Smith DE. A comparison of pullout strength for pedicle screws of different designs: a study using tapped and untapped pilot holes. Spine (Phila Pa 1976) 2006;31 (23):E867-E870 